\title{
ESTUDO DA BIOSSORÇÃO DO CROMO III UTILIZANDO A MACRÓFITA AQUÁTICA SALVINIA AURICULATA
}

\author{
A. P. OLIVEIRA ${ }^{2 *}$, A. N. MÓDENES ${ }^{1}$, M. E. BRAGIÃO ${ }^{1}$, I. G. O. BEZERRA ${ }^{1}$, SAUSEN, M. G. ${ }^{1}$, \\ R. BERGAMASCO ${ }^{2}$ \\ ${ }^{1}$ Universidade Estadual do Oeste do Paraná, Departamento de Engenharia Química \\ ${ }^{2}$ Universidade Estadual de Maringá, Departamento de Engenharia Química \\ e-mail: anaoliveiraeq@gmail.com
}

\begin{abstract}
RESUMO
Neste trabalho foi avaliado o potencial de biossorção da macrófita aquática Salvinia auriculata na remoção de íons Cr III. Foi utilizada a biomassa seca à $40^{\circ} \mathrm{C}$ (in natura) e submetida a tratamento químico com $\mathrm{H}_{3} \mathrm{PO}_{4} 1 \mathrm{~mol} \mathrm{~L}^{-1}$ e $\mathrm{NaOH} 0,1 \mathrm{~mol} \mathrm{~L}{ }^{-1}$. Os ensaios cinético e de equilíbrio foram realizados em sistema batelada sob agitação constante, com $\mathrm{pH}$ e temperatura controlados. Os resultados obtidos para a cinética mostraram que, para a biomassa in natura o tempo de equilíbrio foi em torno de 6 horas, enquanto que para a biomassa tratada o equilíbrio foi atingido com aproximadamente 24 horas, ambos seguindo o modelo cinético de pseudosegunda ordem. No estudo do equilíbrio, verificou-se que o modelo de Langmuir foi o que melhor se ajustou aos dados experimentais para todas a temperaturas de operação avaliadas. $\mathrm{Na}$ temperatura de $30^{\circ} \mathrm{C}$, a capacidade máxima de sorção da $S$. auriculata in natura foi de $8,2 \pm 0,2 \mathrm{mg} \mathrm{g}^{-1}$, com melhoria significativa na eficiência de remoção quando aplicado o tratamento da biomassa, sendo a capacidade de remoção elevada para $22,8 \pm 0,5 \mathrm{mg} \mathrm{g}^{-1}$. Os resultados obtidos demonstram que a macrófita $S$. auriculata tem potencial para ser utilizada em sistemas de tratamento de efluentes contendo íons Cr III.
\end{abstract}

\section{INTRODUÇÃO}

O desenvolvimento de tecnologias alternativas para remoção de poluentes como os metais pesados de águas contaminadas tem se apresentado de significativo interesse ambiental devido à crescente poluição do meio ambiente (PIETROBELLI et al., 2009; ABDEL-GHANI e EL-CHAGHABY, 2014). A contaminação de ambientes aquáticos por estes poluentes pode causar efeitos nocivos à saúde humana devido à sua toxicidade e persistência no meio, acumulando-se ao longo da cadeia alimentar (SOUZA et al., 2013).

Dentre os metais que recebem maior atenção, podemos citar o cromo, frequentemente encontrado em águas residuais sob o estado de oxidação trivalente. Nesta espécie, o cromo apresenta-se como um micronutriente essencial para os organismos vivos quando em baixas concentrações, entretanto, a exposição excessiva ao metal pode ocasionar problemas aos seres humanos por apresentar caráter carcinogênico, entre outros efeitos adversos (DIVYASREE, BRAUN e SUBRAMANIAN, 2014; KANAGARAJ et al., 2014).

O cromo trivalente (Cr III) é usualmente encontrado em águas residuais de indústrias de galvanoplastia, tintas e pigmentos, curtimento de couro, cerâmica e vidro, além de empregos na preservação da madeira (NETZAHUATL-MUÑOZ et al., 2012; YANG et al., 2013). 
No intuito de preservar o ambiente receptor destes efluentes, várias tecnologias de tratamento têm sido empregadas para remover o cromo de águas residuais (SULTANA et al., 2014). Os principais métodos incluem a precipitação (GOLBAZ et al., 2014), eletrocoagulação (MÓDENES et al., 2012), separação por membranas (HABIBI et al., 2015), troca iônica (GONZALEZ et al., 2015), adsorção (REDDY, LAKSHMIPATHY e SARADA, 2014), entre outros. Entre estes métodos, a adsorção apresenta-se como um método com fácil concepção de operação e eficaz (MÓDENES et al., 2015). No entanto, adsorventes comerciais como o carvão ativado, limitam a aplicabilidade deste processo pelo seu elevado custo. $\mathrm{Na}$ atualidade, a biossorção têm recebido grande destaque, com a proposta de diferentes biomassas de baixo custo como potenciais adsorventes de metais pesados presentes em resíduos aquosos. Dentre estes biomateriais podemos citar as algas (SOUZA et al., 2013), bactérias (DIVYASREE,BRAUN e SUBRAMANIAN, 2014; KANAGARAJ et al., 2014), bagaço de cana de açúcar (YANG et al., 2013), casca de soja (MÓDENES et al., 2015), macrófitas aquáticas (MÓDENES et al., 2013) dentre outros resíduos biológicos.

Neste âmbito, o objetivo deste estudo foi avaliar a utilização da macrófita aquática Salvinia auriculata na biossorção dos íons $\mathrm{Cr}$ III, em meio aquoso, por meio de estudos de sorção em batelada. Para tanto, realizou-se o estudo do efeito de parâmetros operacionais como $\mathrm{pH}$, granulometria e temperatura no processo de biossorção, bem como a obtenção de dados cinéticos e de equilíbrio. Foi verificada ainda a aplicabilidade de tratamentos químicos na macrófita, submetendo a biomassa a tratamentos com ácido fosfórico e hidróxido de sódio em diferentes concentrações. Com base na melhor resposta dos tratamentos químicos em termos de capacidade de remoção, foram realizados todos os ensaios preliminares, cinéticos e de equilíbrio com a biomassa modificada. Os dados cinéticos foram modelados utilizando modelos como de pseudoprimeira ordem e pseudosegunda ordem e Elovich, enquanto que isotermas como de Langumuir, Freundlich, Redlich-Peterson, Temkin, Toth, Sips foram testadas, avaliando-se os modelos podem descrever adequadamente o processo.

\section{MATERIAIS E MÉTODOS}

\subsection{Preparo da Biomassa}

A macrófita Salvinia auriculata foi coletada em lagoas na região Oeste do Paraná-Brasil. A planta foi lavada com água corrente, posteriormente, com água destilada, e seca à $40^{\circ} \mathrm{C}$. Em seguida, a biomassa foi triturada e separadas frações de granulometria entre $0,1-1,4 \mathrm{~mm}$.

\subsection{Tratamento Químico da $S$. auriculata}

Para o tratamento químico foram utilizadas soluções de ácido fosfórico $\left(\mathrm{H}_{3} \mathrm{PO}_{4}\right)$ e hidróxido de sódio $(\mathrm{NaOH})$. No tratamento ácido, $10 \mathrm{~g}$ de biomassa moída, na granulometria de 0,1-1,4 mm, foi adicionada em $1 \mathrm{~L}$ de solução de $\mathrm{H}_{3} \mathrm{PO}_{4}$ nas concentrações de 0,05 a $1 \mathrm{~mol} \mathrm{~L}^{-1}$. A mistura foi mantida sob agitação e aquecimento ( 80 ${ }^{\circ} \mathrm{C}$ ) durante 30 minutos, sendo em seguida seca à $100{ }^{\circ} \mathrm{C}$. Enquanto que, no tratamento básico foram utilizadas soluções de $\mathrm{NaOH}$ nas concentrações de 0,1 e $1 \mathrm{~mol} \mathrm{~L}^{-1}$, e a biomassa tratada à temperatura ambiente $\mathrm{e}$ seca à $40^{\circ} \mathrm{C}$. Após a secagem, para ambos os tratamentos, a biomassa foi lavada com água destilada (até ser atingido o $\mathrm{pH}$ neutro na água de lavagem) e seca à $40{ }^{\circ} \mathrm{C}$. Após o tratamento ácido, parte da biomassa tratada nas concentrações de 0,1 e $1 \mathrm{~mol} \mathrm{~L}^{-1}$, foi submetida ao tratamento básico.

\subsection{Solução do Íon Cromo III}

A solução sintética do íon $\mathrm{Cr}$ (III) foi preparada a partir de Nitrato de cromo III 
$\left(\mathrm{Cr}\left(\mathrm{NO}_{3}\right)_{3} .9 \mathrm{H}_{2} \mathrm{O}\right)$ de grau analítico, sendo feita a dissolução em água ultra pura (Milli-Q Plus ${ }^{\circledR}, \quad$ Millipore), na concentração de aproximadamente $140 \mathrm{mg} \mathrm{L}^{-1}$. O pH inicial da solução foi ajustado para 3,0 com soluções de $\mathrm{NaOH}\left(1 \mathrm{~mol} \mathrm{~L}^{-1}\right)$ e $\mathrm{HCl}\left(1 \mathrm{~mol} \mathrm{~L}^{-1}\right)$.

\subsection{Diagrama de Especiação}

O programa MEDUSA acoplado ao HYDRA foi utilizado para avaliação das espécies de Cr III formadas em solução, com base na variação de $\mathrm{pH}$. Construiu-se o diagrama de especiação do metal com base nas constantes de equilíbrio das espécies.

\subsection{Análise AAS}

A concentração do íon cromo, na fase líquida, foi determinada pela técnica de espectroscopia de absorção atômica (AAS) operando com chama ar-acetileno (Modelo AAnalyst 700, Marca PerkinElmer), no comprimento de onda de $357,9 \mathrm{~nm}$. As amostras foram diluídas com $\mathrm{HNO}_{3} 2 \%$.

\subsection{Testes Preliminares}

Foram realizados testes preliminares com diferentes valores de $\mathrm{pH}$ inicial da solução (1 à 5), temperatura de sorção $(20,30$ e $\left.40{ }^{\circ} \mathrm{C}\right)$ e tamanho de partícula $(0,1-0,18$ $\mathrm{mm}, \quad 0,18-1,4 \mathrm{~mm},>1,4 \mathrm{~mm}$, e mistura granulométrica). Além disso, realizou-se testes de adsorção para verificar o efeito dos tratamentos químicos sobre a capacidade de sorção de metal pela $S$. auriculata. Para cada teste, $50 \mathrm{~mL}$ de solução de $\mathrm{Cr}$ III na concentração de $140 \mathrm{mg} \quad \mathrm{L}^{-1}$ foram adicionados a $300 \mathrm{mg}$ de $S$. auriculata devidamente preparada. Os ensaios foram realizados em duplicata. A mistura foi mantida sob agitação de $90 \mathrm{rpm}$ durante 24 horas, sob temperatura controlada. Em seguida, as amostras foram filtradas (membrana de 0,45 $\mu \mathrm{m}$ ), e determinada a concentração do metal da fase líquida por análise em AAS. A capacidade de adsorção foi determinada pelo balanço de massa expresso na Equação 1.
$q=\frac{\left(C-C_{0}\right) V}{m}$

sendo q $\left(\mathrm{mg} \mathrm{g}^{-1}\right)$ é a quantidade de metal adsorvido, $\mathrm{C}_{0}$ e $\mathrm{C}\left(\mathrm{mg} \mathrm{L}^{-1}\right)$ concentrações iniciais e finais de metal em solução, respectivamente, $\mathrm{V}$ (L) é o volume da solução e m (g) é a massa de adsorvente.

\subsection{Ensaios Cinéticos do Íon Cr III}

Com base nas condições definidas nos ensaios preliminares, foram realizados ensaios em pH 3,0 com a adição de $300 \mathrm{mg}$ do biossorvente em $50 \mathrm{~mL}$ de uma solução de $\mathrm{Cr}$ III com concentração inicial de aproximadamente $140 \mathrm{mg} \mathrm{L}^{-1}$. A mistura foi mantida na temperatura de $30{ }^{\circ} \mathrm{C}$, sob agitação constante $(90 \mathrm{rpm})$, sendo utilizada a macrófita moída na fração granulométrica entre 0,1-1,4 mm, com ensaios em duplicata. Retirou-se amostras com tempos de contato entre 5 e 1800 minutos, sendo estas subsequentemente filtradas em membranas de $0,45 \mu \mathrm{m}$ e determinada a concentração residual de metal na fase líquida por AAS. Todos os ensaios foram realizados para a $S$. auriculata in natura e tratada com $\mathrm{H}_{3} \mathrm{PO}_{4} 1$ mol $\mathrm{L}^{-1}$ e $\mathrm{NaOH}$ 0,1 mol $\mathrm{L}^{-1}$. Os dados obtidos nos ensaios cinético, foram modelados por modelos cinéticos amplamente aplicados neste tipo de sistema, como os modelos de pseudoprimeira ordem, pseudossegunda ordem e Elovich.

\subsection{Ensaios de Equilíbrio do Íon Cr III}

Os ensaios de equilíbrio foram realizados frente as condições definidas nos testes preliminares, com tempo de contato de 24 horas. Os testes de equilíbrio em batelada foram feitos prosseguindo-se a mistura de 300 $\mathrm{mg}$ de $S$. auriculata (in natura ou tratada) em $50 \mathrm{~mL}$ de solução de $\mathrm{Cr}$ III, com a concentração da solução variando de 5 a 320 $\mathrm{mg} \mathrm{L}^{-1}$. Os experimentos foram realizados sob agitação constante $(90 \mathrm{rpm})$ e temperatura controlada $\left(30^{\circ} \mathrm{C}\right)$, com $\mathrm{pH}$ inicial da solução 
ajustado em 3,0. Após atingido o tempo de equilíbrio, as fases líquida e sólida foram separadas por filtração à vácuo com membranas de $0,45 \mu \mathrm{m}$. A concentração de metal na fase líquida, antes e após o processo, foi determinada por AAS, sendo estimada a capacidade de remoção do metal pela Equação 1. Dentre as isotermas empregadas para interpretar os dados de equilíbrio citamse os modelos de Langmuir, Freundlich, Reddlich-Peterson, Temkin, Sips e Toth.

\section{RESULTADOS E DISCUSSÕES}

\subsection{Testes Preliminares}

Considerando a significativa influência do $\mathrm{pH}$ da solução nas espécies do metal que apresentam-se disponíveis ao processo de adsorção, foi previsto com auxílio do programa Hydra-Medusa, a distribuição das espécies de Cr III no meio aquoso, conforme apresentado na Figura 1.

Figura 1 - Distribuição das espécies de Cr (III) em função do pH da solução.

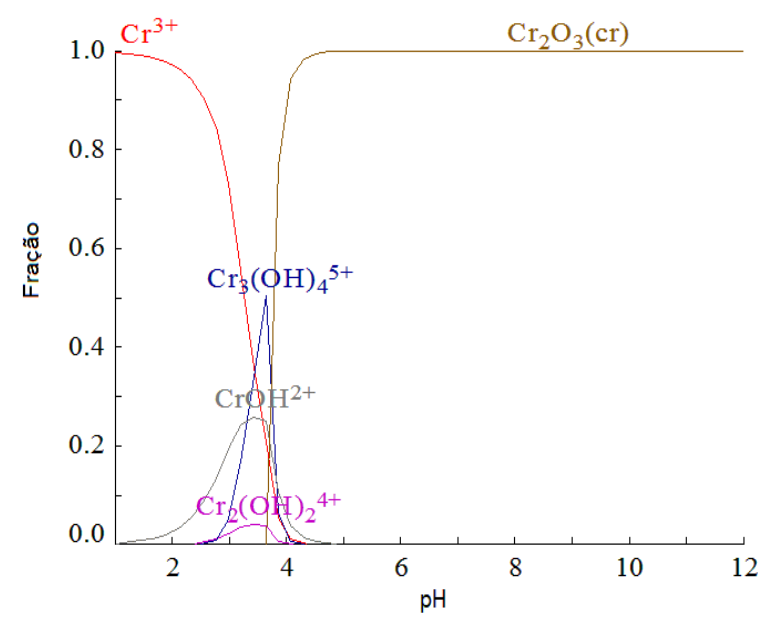

Como observado no diagrama de especiação (Figura 1), para valores de $\mathrm{pH}$ inferiores a 3,0 a espécie predominante consta no $\mathrm{Cr}^{3+}$. Para a faixa entre $\mathrm{pH} 3,0$ e 4,0, as espécies mais abundantes em solução constam em $\mathrm{Cr}^{3+}, \mathrm{Cr}(\mathrm{OH})^{2+}$ e $\mathrm{Cr}_{3}(\mathrm{OH})_{4}{ }^{5+}$. Em pHs superiores a 4,0 , observa-se que o cromo começa a precipitar sob a forma de $\mathrm{Cr}_{2} \mathrm{O}_{3}$.
Tendo em vista a importância do controle do $\mathrm{pH}$ da solução no processo de adsorção, apresenta-se os resultados obtidos para avaliação da influência deste na capacidade de sorção do metal (Tabela 1).

Tabela 1- Influência do $\mathrm{pH}$ da solução na capacidade de remoção dos íons $\mathrm{Cr}$ III $(\mathrm{pH}=3,0$, $\left.\mathrm{T}=30{ }^{\circ} \mathrm{C},[\mathrm{Cr} \mathrm{III}]_{\text {inicial }} \approx 140 \mathrm{mg} \mathrm{L}^{-1}\right)$.

\begin{tabular}{ccccccc}
\hline \multirow{2}{*}{$\mathbf{p H}$} & \multicolumn{2}{c}{ In natura } & \multicolumn{2}{c}{ Tratada } \\
\cline { 2 - 7 } inicial & $\mathbf{p H}$ & \multicolumn{2}{c}{$\mathbf{q H}_{\text {final }}\left(\mathbf{m g ~ g}^{-\mathbf{1}}\right)$} & $\mathbf{p H}$ final & \multicolumn{2}{c}{$\mathbf{q}_{\text {eq }}\left(\mathbf{m g ~ g}^{-\mathbf{1}}\right)$} \\
\hline 1 & 0,92 & $0,71 \pm 0,28$ & 1,07 & $2,48 \pm 0,09$ \\
2 & 2,22 & $1,6 \pm 0,7$ & 2,45 & $4,90 \pm 0,06$ \\
3 & 3,43 & $5,9 \pm 0,4$ & 4,21 & $20,70 \pm 0,06$ \\
4 & 3,95 & $8,3 \pm 0,4$ & 4,91 & $17,1 \pm 0,2$ \\
5 & 4,46 & $10,51 \pm 0,04$ & 5,14 & $9,94 \pm 0,01$ \\
\hline
\end{tabular}

De acordo com os resultados apresentados na Tabela 1, verificar-se que o aumento do $\mathrm{pH}$ do meio favorece significativamente a capacidade de remoção do Cr III pela $S$. auriculata in natura e tratada $\left(\mathrm{H}_{3} \mathrm{PO}_{4} 1\right.$ mol L ${ }^{-1}$ e $\left.\mathrm{NaOH} 0,1 \mathrm{~mol} \mathrm{~L}^{-1}\right)$. Porém, para a planta tratada, a condição de melhor remoção consistiu em $\mathrm{pH} 3,0$, sendo a captura do metal gradativamente reduzida quando avaliados os pHs 4,0 e 5,0.

Entretanto, pelo diagrama de especiação do metal (Figura 1), para valores de $\mathrm{pH}$ superiores a 4,0 pode ocorrer a precipitação do metal. Dessa forma, a condição de trabalho escolhida tanto para a biomassa in natura, como tratada, consistiu em $\mathrm{pH}$ 3,0. Os ensaios em $\mathrm{pH}$ 3,0 resultaram na remoção de $\mathrm{Cr}$ de aproximadamente $27 \%$ para a $S$. auriculata in natura e $88 \%$ para a planta tratada.

Outro parâmetro operacional avaliado consistiu na temperatura do meio, sendo testadas temperaturas de 20,30 e $40^{\circ} \mathrm{C}$, fixando-se as demais condições $(\mathrm{pH}=3,0,90$ rpm, $[\mathrm{Cr} \mathrm{III}]_{\text {inicial }} \approx 140 \mathrm{mg}^{-1}$ ). Foi observado, tanto para a $S$. auriculata in natura como tratada, que a taxa de remoção do metal não foi significativamente influenciada pela temperatura do meio, sendo a variação 
observada inferior a $10 \%$. A capacidade de remoção para a biomassa in natura apresentou-se em torno de $7 \mathrm{mg} \mathrm{g}^{-1}$ e para a planta submetida a tratamento químico, cerca de $18 \mathrm{mg} \mathrm{g}^{-1}$, em ambas as temperaturas avaliadas. Dessa forma, a temperatura escolhida para a realização dos ensaios foi a temperatura ambiente $\left(30^{\circ} \mathrm{C}\right)$.

Por conseguinte, no teste da influência da granulometria na biossorção, para o íon $\mathrm{Cr}$ III pela $S$. auriculata in natura, verificou-se que a taxa de remoção é inversamente proporcional ao tamanho de partícula da biomassa. Os resultados obtidos mostraram que a menor faixa granulométrica testada $(0,1-0,18 \mathrm{~mm})$ proporciona uma capacidade de remoção de $6,9 \pm 0,3 \mathrm{mg} \mathrm{g}^{-1}$, seguida da fração de 0,18-1,4 mm com remoção de 5,50 $\pm 0,13 \mathrm{mg} \mathrm{g}^{-1}$, enquanto que partículas $>1,4$ $\mathrm{mm}$ resultaram em uma leve redução na remoção do metal $\left(5,00 \pm 0,02 \mathrm{mg} \mathrm{g}^{-1}\right)$. Para a fração composta pela mistura granulométrica, a capacidade de remoção do metal apresentou-se intermediária as demais frações $\left(6,6 \pm 0,3 \mathrm{mg} \mathrm{g}^{-1}\right)$. Dessa forma, optou-se pelo emprego da mistura granulométrica nos demais ensaios realizados neste estudo.

\subsection{Tratamento Químico da S. auriculata}

$\mathrm{Na}$ Tabela 2, são apresentados os resultados de capacidade de remoção dos íons $\mathrm{Cr}$ III para os diferentes tratamentos avaliados, utilizando-se $\mathrm{H}_{3} \mathrm{PO}_{4}$ e $\mathrm{NaOH}$.

Observa-se na Tabela 2, que tratamento com $\mathrm{H}_{3} \mathrm{PO}_{4}$ reduz significativamente a taxa de remoção do metal, para todas as concentrações avaliadas. Em contra partida, a planta impregnada com $\mathrm{NaOH}$, apresentou-se mais eficiente na remoção de Cr III, quando comparada a $S$. auriculata in natura, com resultados semelhantes para ambas as concentrações testadas.

Conforme apresentado na Tabela 2, foi avaliada ainda a combinação dos tratamentos ácido e básico, sendo realizado primeiramente o tratamento com $\mathrm{H}_{3} \mathrm{PO}_{4}$ em diferentes
Tabela 2 - Avaliação da capacidade de remoção dos íons Cr III em função de tratamentos químicos na $S$. auriculata. Condições: $\mathrm{pH}=3,0, \mathrm{~T}=30^{\circ} \mathrm{C}$, Agitação $=90 \mathrm{rpm}$.

\begin{tabular}{|c|c|}
\hline Tratamento & $\mathrm{q}_{\mathrm{eq}}\left(\mathrm{mg} \mathrm{g}^{-1}\right)$ \\
\hline In natura & $7,6 \pm 0,2$ \\
\hline $\mathrm{H}_{3} \mathrm{PO}_{4} 0,05 \mathrm{~mol} \mathrm{~L}^{-1}$ & $2,7 \pm 0,3$ \\
\hline $\mathrm{H}_{3} \mathrm{PO}_{4} 0,1 \mathrm{~mol} \mathrm{~L}^{-1}$ & $3,42 \pm 0,08$ \\
\hline $\mathrm{H}_{3} \mathrm{PO}_{4} 0,5 \mathrm{~mol} \mathrm{~L}^{-1}$ & $4,1 \pm 0,1$ \\
\hline $\mathrm{H}_{3} \mathrm{PO}_{4} 1 \mathrm{~mol} \mathrm{~L}^{-1}$ & $2,88 \pm 0,03$ \\
\hline $\mathrm{NaOH} 0,1 \mathrm{~mol} \mathrm{~L}^{-1}$ & $10,5 \pm 0,2$ \\
\hline $\mathrm{NaOH} 1 \mathrm{~mol} \mathrm{~L}^{-1}$ & $10,3 \pm 0,4$ \\
\hline $\mathrm{H}_{3} \mathrm{PO}_{4} 0,1 \mathrm{~mol} \mathrm{~L}^{-1}+\mathrm{NaOH} 0,1 \mathrm{~mol} \mathrm{~L}^{-1}$ & $12,3 \pm 0,3$ \\
\hline $\mathrm{H}_{3} \mathrm{PO}_{4} 0,1 \mathrm{~mol} \mathrm{~L}^{-1}+\mathrm{NaOH} 1 \mathrm{~mol} \mathrm{~L}^{-1}$ & $12,77 \pm 0,02$ \\
\hline $\mathrm{H}_{3} \mathrm{PO}_{4} 1 \mathrm{~mol} \mathrm{~L}^{-1}+\mathrm{NaOH} 0,1 \mathrm{~mol} \mathrm{~L}^{-1}$ & $20,56 \pm 0,07$ \\
\hline $\mathrm{H}_{3} \mathrm{PO}_{4} 1 \mathrm{~mol} \mathrm{~L}^{-1}+\mathrm{NaOH} 1 \mathrm{~mol} \mathrm{~L}^{-1}$ & $21,82 \pm 0,01$ \\
\hline
\end{tabular}

concentrações $\left(0,1\right.$ e $\left.1 \mathrm{~mol} \mathrm{~L}^{-1}\right)$, e subsequente tratamento com $\mathrm{NaOH}$ nas concentrações de 0,1 e $1 \mathrm{~mol} \mathrm{~L}^{-1}$. Para todas as combinações avaliadas, observou-se melhora na capacidade de remoção do $\mathrm{Cr}$ III. Os tratamentos em que utilizou-se $\mathrm{H}_{3} \mathrm{PO}_{4} 1 \mathrm{~mol} \mathrm{~L}^{-1}$, combinados com $\mathrm{NaOH} 0,1$ e $1 \mathrm{~mol} \mathrm{~L}^{-1}$, recebem destaque frente a melhora significativa na capacidade de sorção, atingindo uma taxa de remoção cerca de três vezes superior a planta in natura.

Dessa forma, além da planta in natura, foi escolhido o tratamento combinado de $\mathrm{H}_{3} \mathrm{PO}_{4} 1 \mathrm{~mol} \mathrm{~L}^{-1}$ e $\mathrm{NaOH} 0,1 \mathrm{~mol} \mathrm{~L}^{-1}$, para estudo do processo de sorção dos íons Cr III.

\subsection{Estudo cinético}

A cinética de biossorção foi avaliada para a $S$. auriculata in natura (Figura 2(a)) e tratada com $\mathrm{H}_{3} \mathrm{PO}_{4} 1 \mathrm{~mol} \mathrm{~L}^{-1}$ e $\mathrm{NaOH} 0,1 \mathrm{~mol}$ $\mathrm{L}^{-1}$ (Figura 2(b)). Os dados experimentais da cinética de sorção do Cr (III) para ambas as formas da biomassa (in natura e tratada), foram analisados empregando-se os modelos cinéticos de pseudoprimeira ordem, pseudossegunda ordem e Elovich, sendo os ajustes apresentados na Figura 2. 
Figura 2 - Cinética da sorção do Cr III pela $S$. auriculata (a) in natura e (b) tratada. Condições: $\mathrm{pH}=3,0 ; \mathrm{T}=30^{\circ} \mathrm{C} ;$ Agitação $=90 \mathrm{rpm} ;[\mathrm{Cr}]_{\text {inicial }}$ $\approx 115 \mathrm{mg} \mathrm{L}^{-1}$.

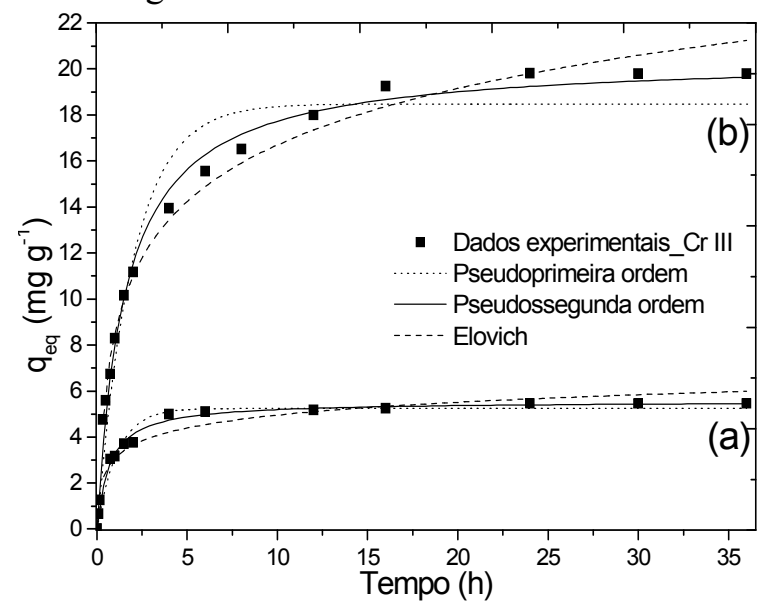

Os dados cinéticos apresentados na Figura 2, demonstraram que a maior parte da remoção do Cr III ocorre nas primeiras horas de contato. Para a biomassa in natura (Figura 2(a)) o tempo de equilíbrio foi atingido após cerca de 6 horas, enquanto que para a biomassa tratada, o equilíbrio se estabeleceu somente em torno de 24 horas (Figura 2(b)). Foi verificado que para a biomassa in natura a capacidade de adsorção aproximou-se de 5 $\mathrm{mg} \mathrm{g}^{-1}$ (30\% de remoção). No entanto, para a biomassa tratada a capacidade de adsorção foi cerca de $20 \mathrm{mg} \mathrm{g}^{-1}$, correspondendo a uma remoção em torno de $80 \%$. Dessa forma, pode-se fazer a indicação da potencialidade de emprego da $S$. auriculata como biossorvente em sistemas de tratamento de efluentes, principalmente quando empregado $\mathrm{o}$ tratamento da biomassa. Em ensaios realizados utilizando-se adsorventes comerciais como o carvão ativado de ossos, mantendo-se as mesmas condições experimentais, a capacidade de adsorção obtida foi de aproximadamente $17 \mathrm{mg} \mathrm{g}^{-1}$.

Os parâmetros dos modelos cinéticos ajustados aos dados experimentais são apresentados na Tabela 3.

Os modelos cinéticos avaliados ajustaram-se aos dados experimentais, sendo observada representatividade do processo pelos mesmos (Tabela 3). Para ambas as formas do adsorvente, verificou-se melhor ajuste pelo modelo de pseudossegunda ordem, com $\mathrm{R}^{2} 0,9925$ para a biomassa in natura, e $\mathrm{R}^{2} 0,9921$ no caso da biomassa tratada.

Tabela 3 - Parâmetros ajustados dos modelos para os dados cinéticos de biossorção do Cr III com a $S$. auriculata in natura e tratada.

\begin{tabular}{|c|c|c|}
\hline \multirow{2}{*}{ Parâmetros } & \multicolumn{2}{|c|}{ S. auriculata } \\
\hline & In natura & Tratada \\
\hline \multicolumn{3}{|c|}{ Modelo de Pseudoprimeira ordem } \\
\hline $\mathrm{q}_{\mathrm{e}}\left(\mathrm{mg} \mathrm{g}^{-1}\right)$ & $5,2 \pm 0,1$ & $18,5 \pm 0,6$ \\
\hline $\mathrm{k}_{1}\left(\mathrm{~h}^{-1}\right)$ & $0,90 \pm 0,09$ & $0,51 \pm 0,06$ \\
\hline $\mathrm{R}^{2}$ & 0,9728 & 0,9536 \\
\hline \multicolumn{3}{|c|}{ Modelo de Pseudossegunda ordem } \\
\hline $\mathrm{q}_{\mathrm{e}}\left(\mathrm{mg} \mathrm{g}^{-1}\right)$ & $5,55 \pm 0,08$ & $20,5 \pm 0,3$ \\
\hline $\mathrm{k}_{2}\left(\mathrm{~g} \mathrm{mg}^{-1} \mathrm{~h}^{-1}\right)$ & $0,26 \pm 0,02$ & $0,031 \pm 0,002$ \\
\hline $\mathrm{R}^{2}$ & 0,9925 & 0,9921 \\
\hline \multicolumn{3}{|c|}{ Modelo de Elovich } \\
\hline $\mathrm{a}\left(\mathrm{mg} \mathrm{g}^{-1} \mathrm{~h}^{-1}\right)$ & $37,4 \pm 12,0$ & $39,4 \pm 4,2$ \\
\hline $\mathrm{b}\left(\mathrm{g} \mathrm{mg}^{-1}\right)$ & $1,24 \pm 0,09$ & $0,282 \pm 0,009$ \\
\hline $\mathrm{R}^{2}$ & 0,9569 & 0,9891 \\
\hline
\end{tabular}

\subsection{Estudo do equilíbrio}

Os dados de equilíbrio, obtidos nas temperaturas de 20,30 e $40^{\circ} \mathrm{C}$, com concentrações iniciais da solução de Cr III variando de 5 à $150 \mathrm{mg} \mathrm{L}^{-1}$ para a $S$. auriculata in natura e entre 5 e $320 \mathrm{mg} \mathrm{L}^{-1}$ para a biomassa tratada. Foram avaliados ainda os principais modelos de isotermas, dentre eles o modelo de Langmuir, Freudlich, Redlich-Peterson, Temkin, Toth e Sips.

Nas Figuras 4 e 5, são apresentados os dados experimentais obtidos para ambas as formas da biomassa, bem como o ajuste da isoterma de Langmuir que melhor representou o processo.

Pode ser observado nas Figuras 4 e 5, que não há variação significativa no comportamento dos dados de equilíbrio em função da variação da temperatura do meio. 
Figura 4 - Isotermas de equilíbrio do Cr III para a $S$. auriculata in natura nas temperaturas de: (a) $\mathrm{T}$ $=20^{\circ} \mathrm{C}$, (b) $\mathrm{T}=30^{\circ} \mathrm{C}$ e (c) $\mathrm{T}=40^{\circ} \mathrm{C}$.
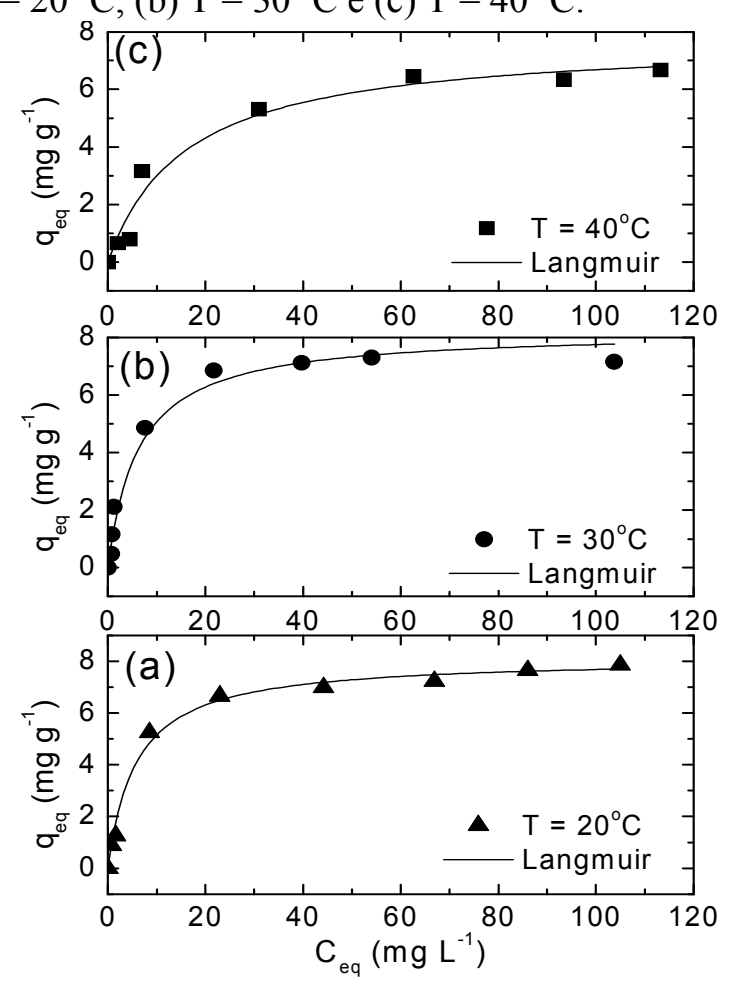

Figura 5 - Isotermas de equilíbrio do Cr III para a $S$. auriculata tratada nas temperaturas de: (a) $\mathrm{T}=$ $20{ }^{\circ} \mathrm{C}$, (b) $\mathrm{T}=30^{\circ} \mathrm{C}$ e (c) $\mathrm{T}=40^{\circ} \mathrm{C}$.

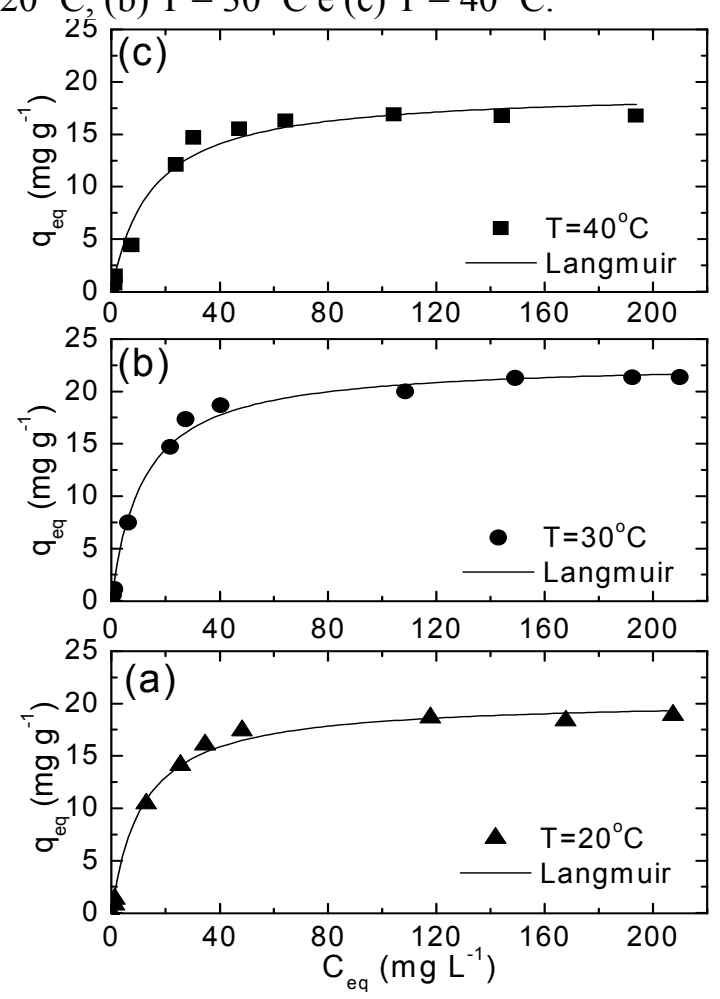

No entanto, para a temperatura de trabalho $\left(30^{\circ} \mathrm{C}\right)$, observou-se valores ligeiramente superiores para a capacidade de adsorção do metal no equilíbrio, pela biomassa in natura, com valores aproximando-se de $8 \mathrm{mg} \mathrm{g}^{-1}$. De forma análoga, para a biomassa tratada, a capacidade máxima de remoção para $30^{\circ} \mathrm{C}$ (cerca de 21 $\mathrm{mg} \mathrm{g}^{-1}$ ) foi levemente superior. Na Tabela 5 são apresentados os parâmetros ajustados para as isotermas de adsorção avaliadas.

Com base na Tabela 5, verifica-se que o modelo que se ajustou melhor aos dados de equilíbrio, foi a isoterma de Langmuir, em ambas as formas da biomassa e temperaturas avaliadas. Dessa maneira, sugere-se que a biossorção ocorre em monocamada, com distribuição homogênea dos sítios de sorção. $\mathrm{Na}$ temperatura de $30^{\circ} \mathrm{C}$, os parâmetros da isoterma de Langmuir para a $S$. auriculata in natura foram $\mathrm{q}_{\max }\left(\mathrm{mg} \mathrm{g}^{-1}\right)$ de $8,2 \pm 0,2 \mathrm{e} \mathrm{b}(\mathrm{L}$ $\mathrm{mg}^{-1}$ ) 0,160 $\pm 0,05$, com $\mathrm{R}^{2}$ de 0,9807. No caso da $S$. auriculata tratada, observou-se a melhora significativa na capacidade de remoção do metal, com $\mathrm{q}_{\max }$ de $22,8 \pm 0,5 \mathrm{e} \mathrm{b}$ $0,086 \pm 0,010$, com $R^{2}$ de 0,9924 . Nos ajustes para os modelos de Redlich-Peterson, Toth e Sips, os parâmetros empíricos admensionais, que indicam a heterogeneidade do adsorvente, $\mathrm{k}$, $\mathrm{n}$, $\mathrm{ns}$, respectivamente, foram iguais a unidade. Dessa forma, os modelos são reduzidos a isoterma de Langmuir.

\subsubsection{Parâmetros termodinâmicos}

As características termodinâmicas do processo de sorção em estudo foram avaliadas em diferentes temperaturas, baseado nas variações de energia livre de Gibbs, entalpia e entropia. A energia livre de Gibbs $\left(\Delta \mathrm{G}^{\circ}\right)$ foi determinada pela Equação 2.

$\Delta G^{0}=-R \ln \left(k_{D}\right)$

em que $\mathrm{R}$ é a constante dos gases ideais $(8,31$ $\left.\mathrm{J} \mathrm{mol}^{-1} \mathrm{~K}^{-1}\right), \mathrm{T}$ a temperatura do meio $(\mathrm{K})$ e $\mathrm{k}_{\mathrm{D}}$ a constante de equilíbrio termodinâmico (L $\mathrm{mg}^{-1}$ ), sendo esta aproximada a constante de 
Tabela 5 - Ajuste dos parâmetros de Isotermas em diferentes temperaturas para o $\mathrm{Cr}$ (III) utilizando a $S$. auriculata in natura e tratada.

\begin{tabular}{|c|c|c|c|c|c|c|c|}
\hline \multirow{2}{*}{$\begin{array}{l}\text { Modelo da } \\
\text { Isoterma }\end{array}$} & \multirow{2}{*}{ Parâmetros } & \multicolumn{3}{|c|}{ S. auriculata in natura } & \multicolumn{3}{|c|}{ S. auriculata tratada } \\
\hline & & $20^{\circ} \mathrm{C}$ & $30^{\circ} \mathrm{C}$ & $40^{\circ} \mathrm{C}$ & $20^{\circ} \mathrm{C}$ & $30^{\circ} \mathrm{C}$ & $40^{\circ} \mathrm{C}$ \\
\hline Langmuir & $\mathrm{q}_{\max }\left(\mathrm{mg} \mathrm{g}^{-1}\right)$ & $8,1 \pm 0,2$ & $8,2 \pm 0,2$ & $7,7 \pm 0,3$ & $20,4 \pm 0,5$ & $22,8 \pm 0,5$ & $19,2 \pm 0,9$ \\
\hline$q_{e}=\frac{q_{\max } b C_{e q}}{2}$ & $\mathrm{~b}\left(\mathrm{~L} \mathrm{mg}^{-1}\right)$ & $0,17 \pm 0,02$ & $0,160 \pm 0,05$ & $0,06 \pm 0,01$ & $0,09 \pm 0,01$ & $0,09 \pm 0,01$ & $0,07 \pm 0,01$ \\
\hline $1+b C_{e q}$ & $\mathrm{R}^{2}$ & 0,9918 & 0,9807 & 0,9697 & 0,9925 & 0,9924 & 0,9772 \\
\hline Freundlich & $\mathrm{k}\left(\mathrm{mg} \mathrm{g}^{-1}\right)$ & $2,2 \pm 0,5$ & $2,2 \pm 0,5$ & $1,1 \pm 0,4$ & $4,8 \pm 1,3$ & $5,1 \pm 1,3$ & $3,9 \pm 1,1$ \\
\hline & $\mathrm{n}$ & $3,4 \pm 0,7$ & $3,4 \pm 0,7$ & $2,5 \pm 0,5$ & $3,6 \pm 0,8$ & $3,5 \pm 0,7$ & $3,2 \pm 0,7$ \\
\hline$q_{e q}=k C_{e q}^{n}$ & $\mathrm{R}^{2}$ & 0,9127 & 0,8800 & 0,9048 & 0,8686 & 0,8836 & 0,8599 \\
\hline Temkin & B & $1,5 \pm 0,1$ & $1,4 \pm 0,1$ & $1,62 \pm 0,15$ & $3,6 \pm 0,2$ & $4,02 \pm 0,25$ & $3,5 \pm 0,3$ \\
\hline$q_{e q}=B \ln \left(k_{T} C_{e q}\right)$ & $\mathrm{k}_{\mathrm{T}}\left(\mathrm{L} \mathrm{mg}^{-1}\right)$ & $2,5 \pm 0,7$ & $3,1 \pm 0,9$ & $0,7 \pm 0,2$ & $1,4 \pm 0,4$ & $1,4 \pm 0,4$ & $1,1 \pm 0,3$ \\
\hline & $\mathrm{R}^{2}$ & 0,9728 & 0,9637 & 0,9590 & 0,9686 & 0,9715 & 0,9468 \\
\hline
\end{tabular}

afinidade obtida pelo ajuste da isoterma de Langmuir. Porém, cabe lembrar que fez-se ainda a aproximação que a constante de atividade é igual a 1. Utilizando-se a equação de van't Hoff, pode-se determinar a variação da entalpia de adsorção $\left(\Delta \mathrm{H}^{\circ}\right)$ e a variação da entropia de adsorção $\left(\Delta \mathrm{S}^{\mathrm{o}}\right)$, relacionando-se estes com $\Delta \mathrm{G}^{\mathrm{o}}$ (Equação 3).

$\ln \left(k_{D}\right)=\frac{\Delta S^{o}}{R}-\frac{\Delta H^{o}}{R T}$

Os parâmetros termodinâmicos determinados para a biossorção dos íons $\mathrm{Cr}$ III, são apresentados na Tabela 6 .

Tabela 6 - Parâmetros termodinâmicos da adsorção dos íons Cr III.

\begin{tabular}{cccc}
\hline T(K) & $\begin{array}{c}\Delta \mathbf{G}^{\mathbf{0}} \\
\left(\mathbf{k J ~ m o l} \mathbf{~}^{-1}\right)\end{array}$ & $\begin{array}{c}\Delta \mathbf{H}^{\mathbf{0}} \\
\left(\mathbf{k J} \mathbf{m o l}^{-1}\right)\end{array}$ & $\begin{array}{c}\Delta \mathbf{S}^{\mathbf{0}} \\
\left(\mathbf{J ~ m o l}^{-1} \mathbf{K}^{-1}\right)\end{array}$ \\
\hline \multicolumn{4}{c}{ S. auriculata in natura } \\
\hline 293,15 & $-22,19$ & & \\
303,15 & $-22,75$ & $-38,24$ & $-53,54$ \\
313,15 & $-21,07$ & & \\
\hline \multicolumn{4}{c}{ S. auriculata tratada } \\
\hline 293,15 & $-20,53$ & & 56,19 \\
303,15 & $-21,11$ & $-4,06$ & \\
313,15 & $-21,65$ & & \\
\hline
\end{tabular}

Com base nos resultados de $\Delta \mathrm{H}^{\mathrm{o}}$ (Tabela 6), pode-se dizer que o processo de adsorção avaliado possui caráter exotérmico, frente aos valores negativos para a planta in natura e tratada, na faixa de temperatura empregada. Além disso, o $\Delta \mathrm{H}^{\circ}$ do processo indica a possibilidade de ocorrer fisiossorção, visto que os valores tanto para a $S$. auriculata in natura, como tratada, apresentam-se na faixa de 0 a $-40 \mathrm{~kJ} \mathrm{~mol}^{-1}$. Apesar do $\Delta \mathrm{H}^{\mathrm{o}}$ indicar processo de adsorção física, no entanto, o processo pode ser coordenado diretamente por grupos funcionais, pela formação de complexos de adsorção na estrutura interna (COELHO et al., 2014). Em relação ao $\Delta \mathrm{G}^{\mathrm{o}}$, verificou-se valores negativos (cerca de $-22 \mathrm{~kJ} \mathrm{~mol}^{-1}$ ), evidenciando um processo espontâneo. Os valores de $\Delta \mathrm{S}^{\mathrm{o}}$ representam a mobilidade associada as interações entre o adsorvente e o adsorbato. Como citado por BERTAGNOLLI et al. (2014), $\Delta S^{o}$ negativos mostram que a reação contribui para diminuir a aleatoriedade da interface adsorvente/adsorbato e a afinidade do biossorvente com o íon metálico. Por outro lado, valores positivos de $\Delta \mathrm{S}^{\mathrm{o}}$ indicam aumento da aleatoriedade e desordem na interface na sorção do metal (COELHO et al., 2014), podendo ainda ocorrer reações de troca iônica (LYUBCHIK et al., 2004).

\section{CONCLUSÕES}

A partir dos resultados dos testes preliminares, foi possível concluir que as melhores taxas de remoção do metal foram 
alcançadas nas condições de $\mathrm{pH}$ superiores a 3,0 , e que a temperatura do meio e o tamanho de partícula do biossorvente não influenciam significativamente na biossorção do metal.

Os resultados obtidos para a cinética de biossorção mostraram que o tempo de equilíbrio é atingido após cerca de 6 horas para a biomassa in natura, enquanto que para a biomassa tratada o equilíbrio se estabelece com aproximadamente 24 horas. Para ambas as formas da biomassa, os dados cinéticos apresentam comportamento que segue $o$ modelo cinético de pseudossegunda ordem. No estudo do equilíbrio, verificou-se que o modelo de Langmuir foi o que melhor se ajustou aos dados experimentais para todas a temperaturas de operação avaliadas, sugerindo que a biossorção dos íons Cr III ocorre em monocamada. A capacidade máxima de biossorção da $S$. auriculata in natura e tratada foi de $8,2 \pm 0,2 \mathrm{mg} \mathrm{g}^{-1}$ e 22,8 $\pm 0,5 \mathrm{mg} \mathrm{g}^{-1}$, respectivamente. Observa-se que o tratamento aumenta quase três vezes a capacidade de remoção do metal. Os parâmetros termodinâmicos determinados, indicaram se tratar de um processo exotérmico e espontâneo, com indícios de fissiossorção. Os resultados obtidos demonstram que a $S$. auriculata tem potencial de emprego em sistemas de tratamento de efluentes contendo íons Cr III, com melhoria significativa na eficiência de remoção quando aplicado o tratamentos da biomassa.

\section{NOMENCLATURA}

AAS - Espectroscopia de Absorção Atômica. [Cr III $]_{\text {inicial }}$ - Concentração inicial da solução de íons Cr III ( $\left.\mathrm{mg} \mathrm{L}^{-1}\right)$.

$\mathrm{T}$ - temperatura $\left({ }^{\circ} \mathrm{C}\right)$

$\mathrm{t}$ - tempo (horas)

\section{REFERÊNCIAS}

ABDEL-GHANI, N.T.; EL-CHAGHABY, G.A.; Biosorption for metal ions removal from aqueous solutions: a review of recent studies; International Journal of Latest Research in Science and Technology; v. 3; n. 1; p. 24-42; 2014.

BERTAGNOLLI, C.; SILVA, M. G. C.; GUIBAL, E.; Chromium biosorption using the residue of alginate extraction from Sargassum filipendula; Chemical Engineering Journal; v.237; p.362-371; 2014.

COELHO, G.F.; GONÇALVES JR., A.C.; TARLEY, C.R.T.; CASARIN, J.; NACKE, H.; FRANCZISKOWSKI, M.A.; Removal of metal ions $\mathrm{Cd}$ (II), $\mathrm{Pb}$ (II), and $\mathrm{Cr}$ (III) from water by the cashew nut shell Anacardium occidentale $\mathbf{L}$; Ecological Engineering; v. 73; p. 514-525; 2014.

DIVYASREE, P.; BRAUN, J.J.; SUBRAMANIAN, S.; Comparative Studies on the Bioremediation of Hexavalent and Trivalent Chromium using Citrobacter freundii: Part I-Effect of parameters controlling Biosorption; Int. J. Environ. Res.; v. 8; n. 4; p. 1127-1134; 2014.

GONZALEZ, M.R.; PEREYRA, A.M.; ZERBINO, R.; BASALDELLA, E.I.; Removal and cementitious immobilization of heavy metals: chromium capture by zeolite-hybridized materials obtained from spent fluid cracking catalysts; Journal of Cleaner Production; v. 91; p. 187-190; 2015.

GOLBAZ, S.; JAFARI, A.J.; RAFIEE, M.; KALANTARY, R.R.; Separate and simultaneous removal of phenol, chromium, and cyanide from aqueous solution by coagulation/precipitation: Mechanisms and theory; Chemical Engineering Journal; v.253; p.251-257; 2014.

HABIBI， S.; NEMATOLLAHZADEH，A.; MOUSAVI, S.A.; Nano-scale modification of polysulfone membrane matrix and the 
surface for the separation of chromium ions from water; Chemical Engineering Journal; v. 267; p. 306-316; 2015.

KANAGARAJ, J.; SENTHILVELAN, T.; PANDA, R.C.; ARAVINDHAN, R.; MANDAL, A.B.; Biosorption of Trivalent Chromium from Wastewater:An Approach towards Green Chemistry; Chem. Eng. Technol.; v. 37; n. 10; p. 1741-1750; 2014.

LYUBCHIK, S.I.; LYUBCHIK, A.I.; GALUSHKO, O.L.; TIKHONOVA, L.P.; VITAL, J.; FONSECA, I.M.; LYUBCHIK, S.B.; Kinetics and thermodynamics of the $\mathrm{Cr}$ (III) adsorption on the activated carbon from co-mingled wastes; Colloids and Surfaces A: Physicochem. Eng. Aspects; v. 242; p. 151-158; 2004.

MÓDENES, A.N.; ESPINOZA-QUIÑONES, F.R.; BORBA, F.H.; MANENTI, D.R.; Performance evaluation of an integrated Photo-Fenton - Electrocoagulation process applied to pollutant removal from tannery effluent in batch system; Chemical Engineering Journal; v. 197; p. 1-9; 2012.

MÓDENES, A.N.; ESPINOZA-QUIÑONES, F.R.; SANTOS, G.H.F.; BORBA, C.E., RIZZUTTO, M.A.; Assessment of metal sorption mechanisms by aquatic macrophytes using PIXE analysis; Journal of Hazardous Materials; v.261; p.148-154; 2013.

MÓDENES, A.N.; ESPINOZA-QUIÑONES, F.R.; COLOMBO, A.; GERALDI, C.L.; TRIGUEROS, D.E.G.; Inhibitory effect on the uptake and diffusion of $\mathrm{Cd}^{2+}$ onto soybean hull sorbent in $\mathrm{Cd}-\mathrm{Pb}$ binary sorption systems; Journal of Environmental Management; v. 154; n. 22-32; 2015.

NETZAHUATL-MUÑOZ, A.R.; GUILLÉNJIMÉNEZ，F.M.; CHÁVEZ-GÓMEZ， B.;
VILLEGAS-GARRIDO, T.L.; CRISTIANIURBINA, E.; Kinetic Study of the Effect of pH on Hexavalent and Trivalent Chromium Removal from Aqueous Solution by Cupressus lusitanica Bark; Water Air Soil Pollut.; v.223; p.625-641; 2012.

PIETROBELLI, J.M.T.A.; MÓDENES, A.N.; FAGUNDES-KLEN, M.R.; ESPINOZAQUIÑONES, F.R.; Cadmium, Copper and Zinc Biosorption Study by Non-Living Egeria densa Biomass; Water Air Soil Pollut; v. 202; p. 385-392; 2009.

REDDY, N. A.; LAKSHMIPATHY, R.; SARADA, N.C.; Application of Citrullus lanatus rind as biosorbent for removal of trivalent chromium from aqueous solution; Alexandria Engineering Journal; v. 53; p. 969-975; 2014.

SOUZA, F. B.; GUELLI ULSON DE SOUZA, S. M. A.; ULSON DE SOUZA, A. A.; COSTA, C. A. E.; BOTELHO, C. M. S.; VILAR, V. J. P.; BOAVENTURA, R. A. R.; Modeling of trivalent chromium speciation in binding sites of marine macroalgae Sargassum Cymosum; Clean Techn Environ Policy; v. 15; p. 987-997; 2013.

SULTANA, M. Y.; AKRATOS C. S.; PAVLOU, S.; VAYENAS, D. V.; Chromium removal in constructed wetlands: A review; International Biodeterioration \& Biodegradation; v. 96; p.181-190; 2014.

YANG, Z.H.; XIONG, S.; WANG, B.; LI, Q.; YANG, W.C.; $\mathbf{C r}$ (III) adsorption by sugarcane pulp residue and biochar; J. Cent. South Univ.; v. 20; p.1319-1325; 2013. 